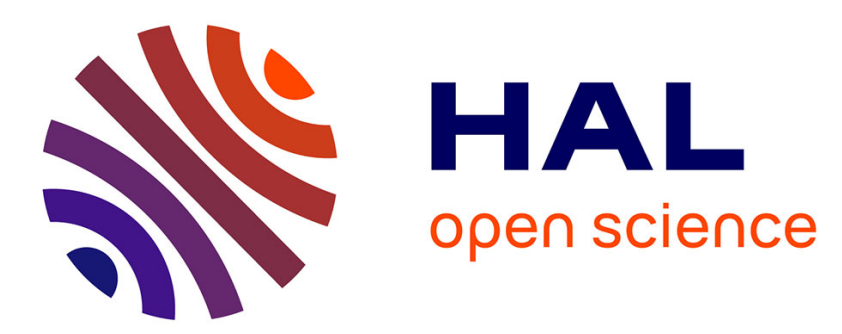

\title{
Diet-induced insulin resistance state disturbs brain clock processes and alters tuning of clock outputs in the Sand rat, Psammomys obesus
}

\author{
Hanane Touati, Saliha Ouali-Hassenaoui, Aicha Dekar-Madoui, Etienne \\ Challet, Paul Pevet, Patrick Vuillez
}

\section{To cite this version:}

Hanane Touati, Saliha Ouali-Hassenaoui, Aicha Dekar-Madoui, Etienne Challet, Paul Pevet, et al.. Diet-induced insulin resistance state disturbs brain clock processes and alters tuning of clock outputs in the Sand rat, Psammomys obesus. Brain Research, 2018, 1679, pp.116-124. 10.1016/j.brainres.2017.11.018 . hal-02348050

\section{HAL Id: hal-02348050 \\ https://hal.science/hal-02348050}

Submitted on 8 Oct 2021

HAL is a multi-disciplinary open access archive for the deposit and dissemination of scientific research documents, whether they are published or not. The documents may come from teaching and research institutions in France or abroad, or from public or private research centers.
L'archive ouverte pluridisciplinaire HAL, est destinée au dépôt et à la diffusion de documents scientifiques de niveau recherche, publiés ou non, émanant des établissements d'enseignement et de recherche français ou étrangers, des laboratoires publics ou privés. 
Research report

\title{
Diet-induced insulin resistance state disturbs brain clock processes and alters tuning of clock outputs in the Sand rat, Psammomys obesus
}

\author{
Hanane Touati $^{\mathrm{a}, \mathrm{b}}$, , Saliha Ouali-Hassenaoui ${ }^{\mathrm{b}}$, Aicha Dekar-Madoui ${ }^{\mathrm{b}}$, Etienne Challet $^{\mathrm{a}}$, Paul Pévet $^{\mathrm{a}}$, \\ Patrick Vuillez ${ }^{\mathrm{a}}$ \\ ${ }^{a}$ Regulation of Circadian Clocks Team, Institute of Cellular and Integrative Neurosciences, UPR3212, CNRS, University of Strasbourg, Strasbourg, France \\ ${ }^{\mathrm{b}}$ USTHB, Faculty of Biological Sciences, Laboratory of Biology and Physiology of Organisms, Neurobiology Team, BP 32, El Alia, 16111 Bab Ezzouar, Algiers, Algeria
}

\section{A R T I CLE IN F O}

Keywords:

Suprachiasmatic nuclei

Vasopressin

CLOCK

Vasoactive intestinal peptide

High-energy diet

Psammomys obesus

\begin{abstract}
A B S T R A C T
Reciprocal interactions closely connect energy metabolism with circadian rhythmicity. Altered clockwork and circadian desynchronization are often linked with impaired energy regulation. Conversely, metabolic disturbances have been associated with altered autonomic and hormonal rhythms. The effects of high-energy (HE) diet on the master clock in the suprachiasmatic nuclei (SCN) remain unclear.This question was addressed in the Sand rat (Psammomys obesus), a non-insulin-dependent diabetes mellitus (NIDDM) animal model. The aim of this work was to determine whether enriched diet in Psammomys affects locomotor activity rhythm, as well as daily oscillations in the master clock of the SCN and in an extra-SCN brain oscillator, the piriform cortex. Sand rats were fed during 3 months with either low or HE diet. Vasoactive intestinal peptide (VIP), vasopressin (AVP) and CLOCK protein cycling were studied by immunohistochemistry and running wheel protocol was used for behavioral analysis. High energy feeding dietary triggered hyperinsulinemia, impaired insulin/glucose ratio and disruption in pancreatic hormonal rhythms. Circadian disturbances in hyper-insulinemic animals include a lengthened rest/activity rhythm in constant darkness, as well as disappearance of daily rhythmicity of VIP, AVP and the circadian transcription factor CLOCK within the suprachiasmatic clock. In addition, daily rhythmicity of VIP and CLOCK was abolished by HE diet in a secondary brain oscillator, the piriform cortex. Our findings highlight a major impact of diabetogenic diet on central and peripheral rhythmicity. The Psammomys model will be instrumental to better understand the functional links between circadian clocks, glucose intolerance and insulin resistance state.
\end{abstract}

\begin{tabular}{|ll|}
\hline \multicolumn{2}{|l|}{ Nomenclature } \\
\multicolumn{2}{|l|}{ Abbreviation } \\
AVP & vasopressin \\
DD & dark/dark \\
HE & high-energy \\
LD & light/dark \\
LE & low-energy \\
NIDDM & non-insulin-dependent diabetes mellitus \\
SCN & suprachiasmatic nuclei \\
VIP & vasoactive intestinal peptide \\
ZT & zeitgeber time \\
\hline
\end{tabular}

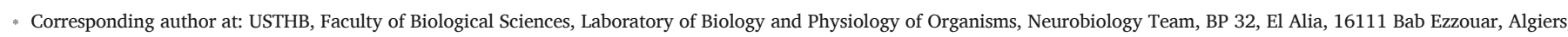
Algeria.

Email addresses: htouati@usthb.dz (H. Touati); souali@usthb.dz (S. Ouali-Hassenaoui); adekar@usthb.dz (A. Dekar-Madoui); challet@inci-cnrs.unistra.fr (E. Challet); pevet@inci-cnrs. unistra.fr (P. Pévet); vuillez@inci-cnrs.unistra.fr (P. Vuillez)

\section{Introduction}

Daily rhythms of sleep/wake and feeding/fasting are controlled by a master hypothalamic clock located in the SCN that is mainly reset by ambient light perceived by the retina (Golombek and Rosenstein, 2010). Secondary circadian clocks and oscillators, such as extra-SCN brain areas or peripheral tissues (e.g., liver and white adipose tissue), are synchronized by the SCN clock and can be influenced by various extracellular signals, in particular those associated with timed feeding. Conversely, the light-entrainable SCN clock is relatively impervious to meal timing. Nonetheless, functioning of the SCN clock is differentially altered by unbalanced energy balance, due to either hypo- or hyper-caloric feeding, thus showing that metabolic cues affect the SCN clock. For instance, independently of timing of feeding, caloric restriction in mice affects the SCN clockwork (Challet, 2010; Sen et al., 2016) and increases light-in- 
duced phase shifts and molecular responses of the SCN (Mendoza et al., 2007). On the other hand, diet-induced obesity in mice leads to lengthened endogenous period (Kohsaka et al., 2007).

Although the metabolic signals underlying these circadian modifications are not clearly identified yet, metabolic hormones such as leptin and insulin are possible candidates because they modulate SCN function. Among others, phase-shifting effects of in vivo leptin have been recently characterized (Mendoza et al., 2011). In vitro application of insulin on hypothalamic slices inhibits the firing rate of SCN neurons (Shibata et al., 1986), an effect likely mediated by insulin receptors expressed in SCN cells (Unger et al., 1989). Insulin injected into the SCN modulates peripheral metabolic cycles, like plasma glucose rhythm through circadian modulation of autonomic nervous system (Mori et al., 1985; Sakaguchi et al., 1988). Both insulin resistance state and diet-induced obesity are associated with flattened circadian autonomic and hormonal rhythms (e.g., Emdin et al., 2001; Kohsaka et al., 2007; Perciaccante et al., 2006) and disruption in peripheral clock oscillations (Kohsaka et al., 2007).

The Sand rat, Psammomys obesus, is a Saharan herbivorous rodent, mainly used as an animal model to study NIDDM (Hackel et al., 1965).

Moreover, when fed with laboratory chow diet (i.e., a diet with higher energy content than their normal diet comprised of low-energy salty bushes), most Psammomys develop NIDDM, including glucose intolerance, obesity and severe insulin resistance, eventually leading to lethal ketonic form (Hackel et al., 1965).

Even if this model of diet-induced NIDDM has been deeply studied to understand NIDDM pathogenesis, it still remains unexplored whether high-energy diet and its diabetogenic consequences could alter cycling of the master clock in the SCN and the piriform cortex as a secondary brain oscillator.

In this study, the difference between the two diets resides in the quantity of fibers and fat. Low-energy diet (LE: $1.8 \mathrm{kcal} / \mathrm{g}$ ), is a high-fiber diet containing four-time higher quantity of fibers than standard chow (HE: $2.50 \mathrm{kcal} / \mathrm{g}$ ), fat proportion is also high for reason that fibers lower fat absorption.

\section{Results}

\subsection{Behavioral and physiological effects of high-energy feeding}

We first examined the changes in metabolic state and locomotor activity rhythm under light/dark (LD) and dark/dark (DD) conditions.

\subsubsection{Changes in body weight and food intake}

Body weight and caloric-intake curves of HE-fed animals $(\mathrm{n}=10)$ and control Psammomys $(\mathrm{n}=12)$ are shown in Fig. 1 . We observed significant effect of both diet and time on the evolution of body weight $(\mathrm{P}=0.04, \mathrm{P}<0.001)$ and caloric intake respectively $(\mathrm{P}=0.011$, $\mathrm{P}<0.001$; two-way repeated measures ANOVA on ranks). A significant interaction was noted for body weight only $(\mathrm{P}<0.001)$.

Wheel access reduced significantly caloric intake during the first week $(48.3 \pm 1.5$ vs. $36.8 \pm 2.6 \mathrm{kcal}$ in control group, $\mathrm{P}<0.01$; and $39.3 \pm 2.9$ vs. $32.2 \pm 3.5 \mathrm{kcal}$ in HE group, $\mathrm{P}<0.05$; paired Student $t$ test). Wheel access induced also diminution of body weight variation during the first week in both HE-fed and control animals, respectively $(8.1 \pm 1.3$ before wheel access vs. $-3.7 \pm 3.3 \mathrm{~g}$ after wheel access, $\mathrm{P}<0.01$, paired Student $\mathrm{t}$ test; and $3.7 \pm 1.1$ before wheel access vs. - $9 \pm 4.2 \mathrm{~g}$ after wheel access; $\mathrm{P}=0.012$; Wilcoxon test).

\subsubsection{Metabolic state}

The day-night variations of non fasting plasma glucose and insulin in HE-fed animals $(\mathrm{n}=10)$ and control Psammomys $(\mathrm{n}=12)$ are shown in Fig. 2A and B. We observed significant effect of diet on insulinemia and ratio variation $(\mathrm{P}<0.001)$. The $\mathrm{HE}$ diet caused significant elevated insulinaemia $(9.3 \pm 0.4$ vs. $3 \pm 0.4 \mu \mathrm{g} / \mathrm{mL}$ at day, $\mathrm{P}<0.01 ; 8.4 \pm 2.1$ vs.

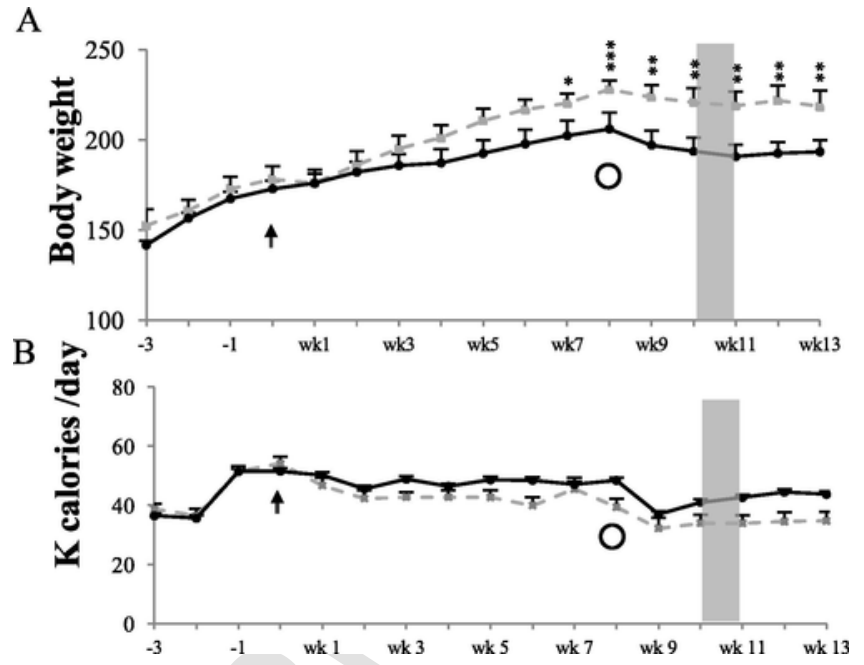

Fig. 1. Changes in body weight (A) and calorie intake (B) in control Psammomys fed with low-energy (LE) diet (dark line, $\mathrm{n}=12$ ), and high-energy (HE) fed animals (gray line, $\mathrm{n}=10$ ). Results are expressed as means \pm SEM, Two-way repeated measures ANOVA on ranks, ${ }^{*} \mathrm{P}<0.05,{ }^{* * *} \mathrm{P}<0.01,{ }^{* * *} \mathrm{P}<0.001$ vs. wk 1 in $\mathrm{HE}$ group. Arrows and circles show the beginning of diet treatment and the introduction of wheel, respectively. Shaded rectangles indicate one week spent in constant darkness (DD). wk, weeks.

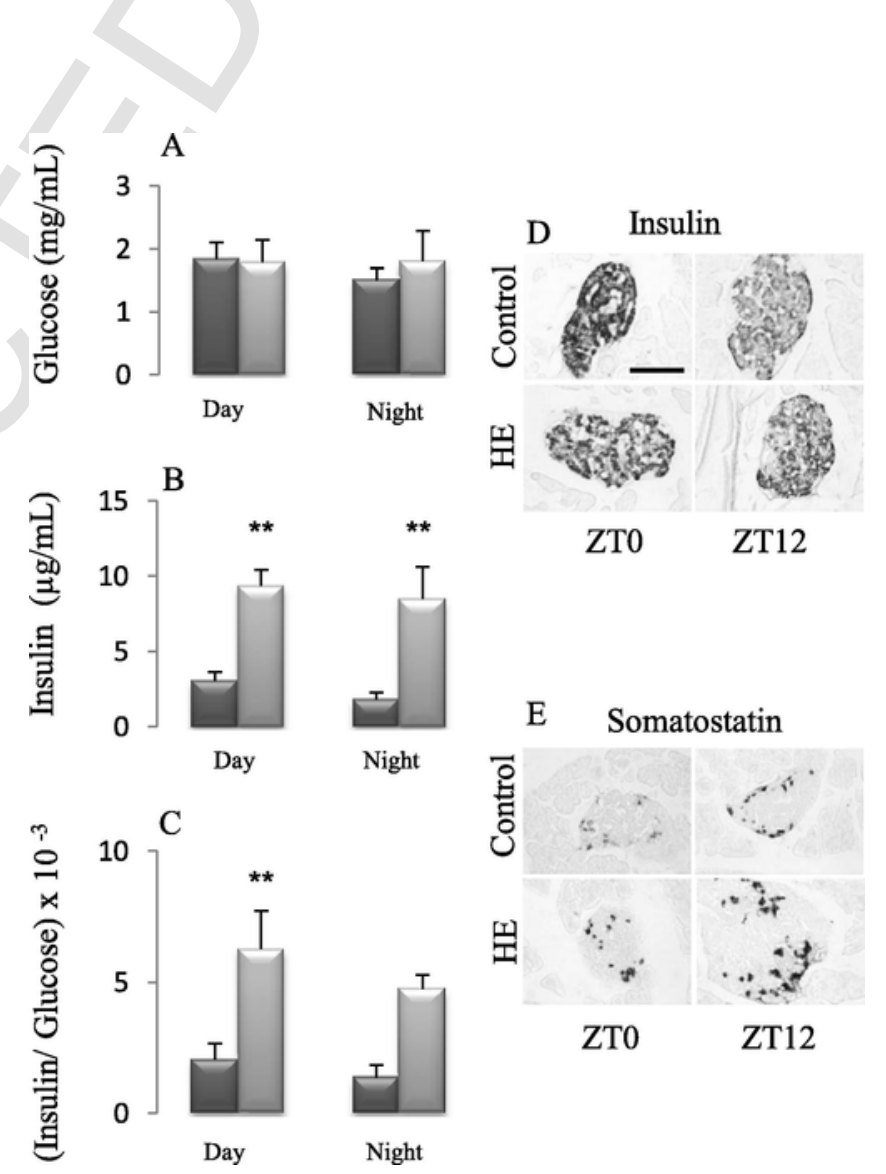

Fig. 2. Effect of high-energy (HE) feeding on plasma parameters and daily pancreatic islets content in Psammomys. Glucose level (A), insulin level (B), insulin/glucose ratio (C). Insulin and glucose profiles were measured during day (ZT 0 and ZT 6) and night (ZT 12 and ZT18) after three months of diet treatment in control Psammomys (dark bars $\mathrm{n}=12$ ), and HE-diet fed Psammomys (gray bars, $\mathrm{n}=10$ ) kept under standard light-dark conditions. Values are mean \pm SEM. Photomicrographs from insulin and somatostatin immunostained sections (D, E). Values are mean \pm SEM, Two-way ANOVA on ranks, ${ }^{* *} \mathrm{P}<0.01$ vs. controls. Scale bar: $100 \mu \mathrm{m}$. 
$1.8 \pm 0.2 \mu \mathrm{g} / \mathrm{mL}$ at night, $\mathrm{P}<0.01)$ and insulin/glucose ratio ( $6.210^{-3} \pm 1.210^{-3}$ vs. $2.110^{-3} \pm 0.510^{-3}$ at day, $\mathrm{P}<0.01$, Fig. $2 \mathrm{C}$ ). Both groups did not show significant daily variations in glycaemia or insulinaemia ( $\mathrm{P}>0.05$; tow-way ANOVA on ranks).

\subsubsection{Day-night variations of pancreatic hormonal content is disturbed}

The day-night profiles of insulin and somatostatin immunoreactivity in Psammomys islets are shown in Fig. 2D and E. There is a significant effect of time $(\mathrm{P}<0.03)$ and diet $(\mathrm{P}<0.03)$ on pancreatic insulin and an effect of time close to significance on pancreatic somatostatin $(\mathrm{P}=0.059)$, with significant interaction for both hormones $(P<0.001)$. In control animals $(n=6)$, insulin and somatostatin immunoreactive signals showed day-night differences with higher values of insulin and somatostatin observed at zeitgeber time (ZT)0 $(\mathrm{P}<0.01)$ and ZT12 $(\mathrm{P}<0.05$; two way ANOVA on ranks), respectively. This tendency is lost in HE-fed animals $(n=6)$, islets insulin content being lower in treated animals at ZT0 (Fig. 2D), while somatostatin immunoreactivity remaining elevated (Fig. 2E) when compared with controls at ZT0. The two profiles progress in antiphase across daytime for each group.

\subsubsection{High-energy diet alters locomotor activity and lengthens the endogenous period}

Controls $(n=12)$ and HE-fed animals $(n=10)$ entrained to LD cycles were rhythmics and showed nocturnal pattern of locomotor activity (Fig. 3A) (80\% of their total activity occurred during the night phase). Nevertheless, HE-fed Psammomys showed reduced levels of locomotor activity (Fig. 3B). The total number of wheel revolutions was significantly reduced $(-45 \%)$ in $\mathrm{LD}$, but only during the active phase (7989 \pm 1067 vs. $14,668 \pm 2176$ revolutions, $\mathrm{P}<0.05$, Kruskal-Wallis test).

\section{A}

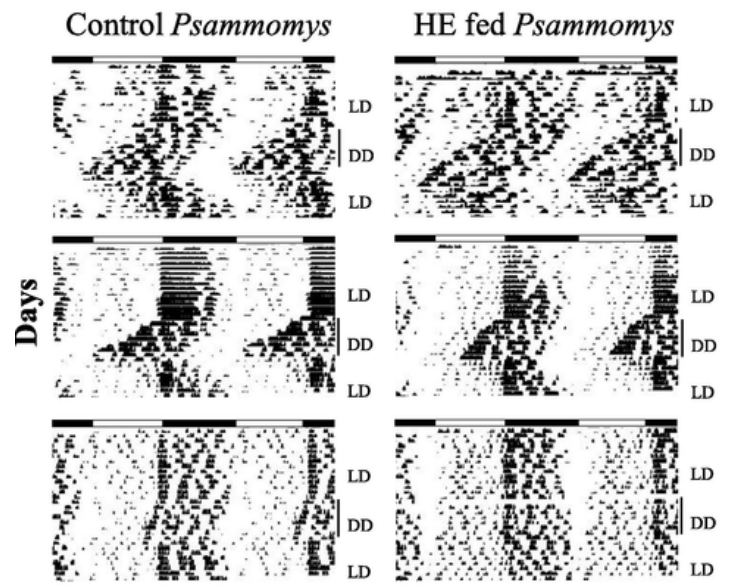

Moreover, a total reduction $(-41 \%)$ was detected in the HE animals housed in DD $(8514 \pm 1899$ vs. $14,372 \pm 1899$ wheel revolutions, $\mathrm{P}<0.05$, Kruskal-Wallis test). We next examined the effect of HE diet on the free-period of wheel-running activity. After the transfer of the two groups to DD for 10 days, HE fed animals expressed a longer free-running period (Fig. 3C) compared to control animals (23.4 \pm 0.2 vs. $22.8 \pm 0.2 ; \mathrm{P}<0.05$, t.test).

\subsection{Effects of high-energy feeding on the SCN and piriform cortex}

\subsubsection{Cytoarchitecture of the SCN in Psammomys}

The SCN in Psammomys (Fig. 4A) are paired nuclei, located in the anterior hypothalamus. In their rostro-caudal extent they are symmetric, separated by the third ventricle, limited dorsally by the periventricular nuclei and ventrally by a thick optic chiasm. Each nucleus is ovoid in its rostral part then appears as a pear-like shape in its medial part (Fig. 4A). At this level, the nucleus extends around $430 \mu \mathrm{m}$ along the dorso-ventral axis and $330 \mu \mathrm{m}$ laterally. More caudally the nucleus presents a circular form and smaller size until its disappearance in the retro-chiasmatic area. Within the nucleus, neurons are densely packed; their mean size is about $11 \mu \mathrm{m}$.

\subsubsection{Immunohistochemical characterization of the SCN}

At dawn (ZT0), the Psammomys SCN shows a classical distribution of AVP in the shell, while VIP and NPY are located in the core region. AVP somata are located dorso-medially in the SCN (Fig. 4B). Most VIP neurons are present in the lower part of the core subdivision (Fig. 4C), while diffuse VIP-positive somas are also found under each SCN within upper

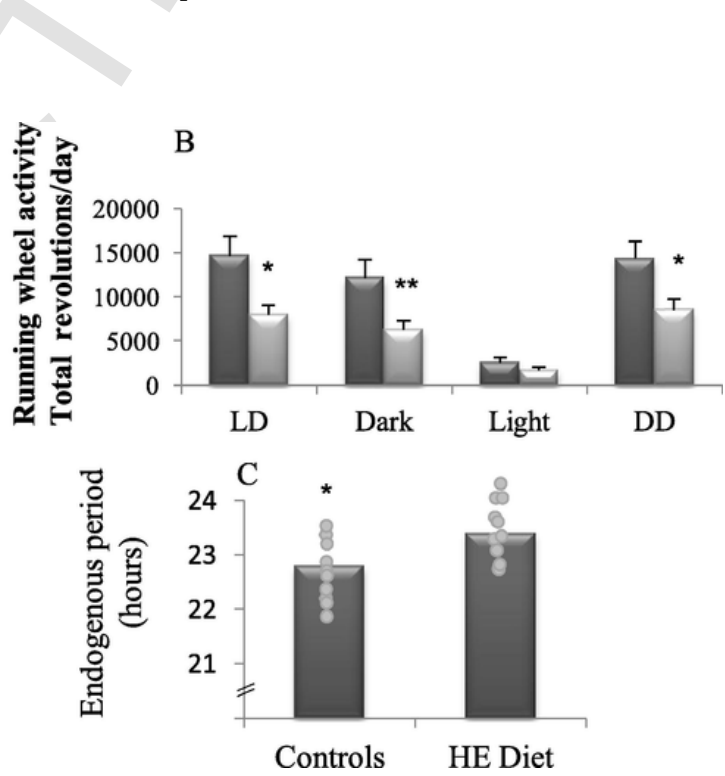

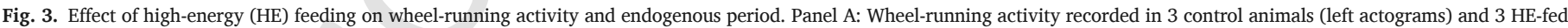

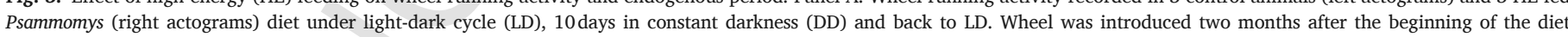

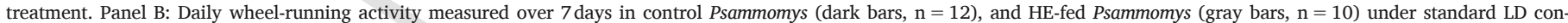

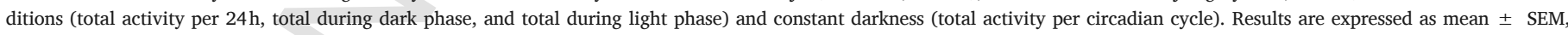

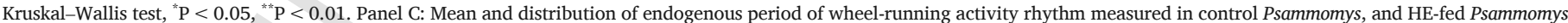
during 8 days of DD (i.e., 10th week of diet treatment), Student t test, " $\mathrm{P}<0.05$.
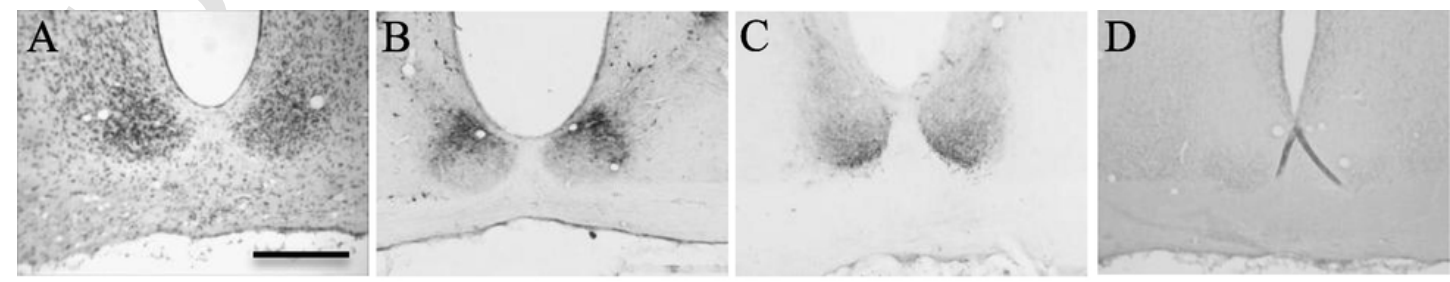

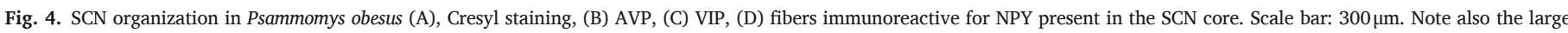
thickness of the optic chiasma under the SCN. 
fibers of the optic chiasm. NPY immunoreactive staining is observed in fibers distributed in the ventral part of the SCN (Fig. 4D).

\subsubsection{Daily oscillations in the SCN}

Two-way ANOVA on ranks (Aligned Rank Transform) indicates significant effect of time on SCN markers (AVP, P $=0.001$ and CLOCK, $\mathrm{P}<0.001$ ) except VIP. The effect of diet was noted on AVP content $(\mathrm{P}=0.009)$, in addition to a significant time $\mathrm{x}$ diet interaction $(P=0.008)$; while the interaction is at the limit of significance for CLOCK $(P=0.057)$, in the absence of significant effect of diet on both VIP and CLOCK. Tukey post hoc tests showed altered rhythmicity of AVP and CLOCK in HE group, with higher AVP content at ZT 18 $(P=0.012)$. This was followed by the post hoc contrast function that reveals a significant difference between the two groups for both AVP $(P=0.01)$ and CLOCK content $(P<0.01)$ during day course [ ZTO - ZT12].

However, cosinor regression revealed significant circadian variation for all markers analysed in the SCN of control animals $(n=12)$.

Acrophases for AVP, VIP and CLOCK occurred close to ZT0, ZT2, and ZT1, respectively (Table 1). By contrast, under HE diet, the cosinor regression of each protein in the SCN did not attain the threshold of significance $(\mathrm{n}=10)$, indicating abolished rhythm for AVP (Fig. 5A and B), VIP (Figure C and D), and CLOCK (Fig. 5E and F). For AVP, lack of daily rhythmicity in HE fed Psammomys was associated with up-regulated levels in the SCN in comparison to control animals (Table 1). VIP and CLOCK levels in the SCN of HE-fed animals were kept at intermediate values compared to those in control animals.

\subsubsection{Daily oscillations in the piriform cortex}

Two-way ANOVA on ranks indicated significant effect of time on VIP $(P=0.02)$ and CLOCK $(P<0.001)$ in the piriform cortex, and significant effect of diet on CLOCK variation $(\mathrm{P}<0.002)$ but not on VIP. Significant interaction between factors was found for CLOCK $(\mathrm{P}=0.047)$ and near to the threshold of significance for VIP $(P=0.063)$. Post hoc testing revealed abolished rhythmicity of CLOCK and VIP in addition to significant lower CLOCK protein level in HE group at ZTO $(P=0.006)$. Tukey post hoc contrast function revealed a significant difference between the two groups for both $\operatorname{CLOCK}(\mathrm{P}=0.006)$ and VIP $(\mathrm{P}=0.01)$ content during day course [

Table 1

Cosinor analysis of AVP, VIP and CLOCK circadian profiles in the suprachiasmatic nuclei and piriform cortex from high energy fed-Psammomys and controls.

\begin{tabular}{|c|c|c|c|c|c|c|}
\hline & \multicolumn{2}{|c|}{ Mean level (A, relative optical density) } & \multicolumn{2}{|c|}{ Amplitude (B, relative optical density) } & \multicolumn{2}{|c|}{ Acrophase $(\mathrm{C}, \mathrm{ZT})$} \\
\hline & $\mathrm{LE}$ & $\mathrm{HE}$ & $\mathrm{LE}$ & $\mathrm{HE}$ & $\mathrm{LE}$ & $\mathrm{HE}$ \\
\hline AVP in SCN & $16.7 \pm 1$ & $21.9 \pm 2.7$ & $7.9 \pm 1.4$ & NS & $0.5 \pm 0.6$ & NS \\
\hline VIP in SCN & $25.37 \pm 4.1$ & $14.9 \pm 3.8$ & $15.7 \pm 5.7$ & NS & $2 \pm 1.4$ & NS \\
\hline CLOCK in SCN & $16.6 \pm 1.6$ & $14.3 \pm 1.3$ & $7.7 \pm 2.3$ & NS & $1.5 \pm 1.1$ & NS \\
\hline VIP in piriform cortex & $2.2 \pm 0.3$ & $1.8 \pm 0.3$ & $1 \pm 0.4$ & NS & $3.1 \pm 1.5$ & NS \\
\hline CLOCK in piriform cortex & $8.1 \pm 0.8$ & $4.9 \pm 1$ & $3.6 \pm 1.2$ & NS & $23.8 \pm 1,2$ & NS \\
\hline
\end{tabular}
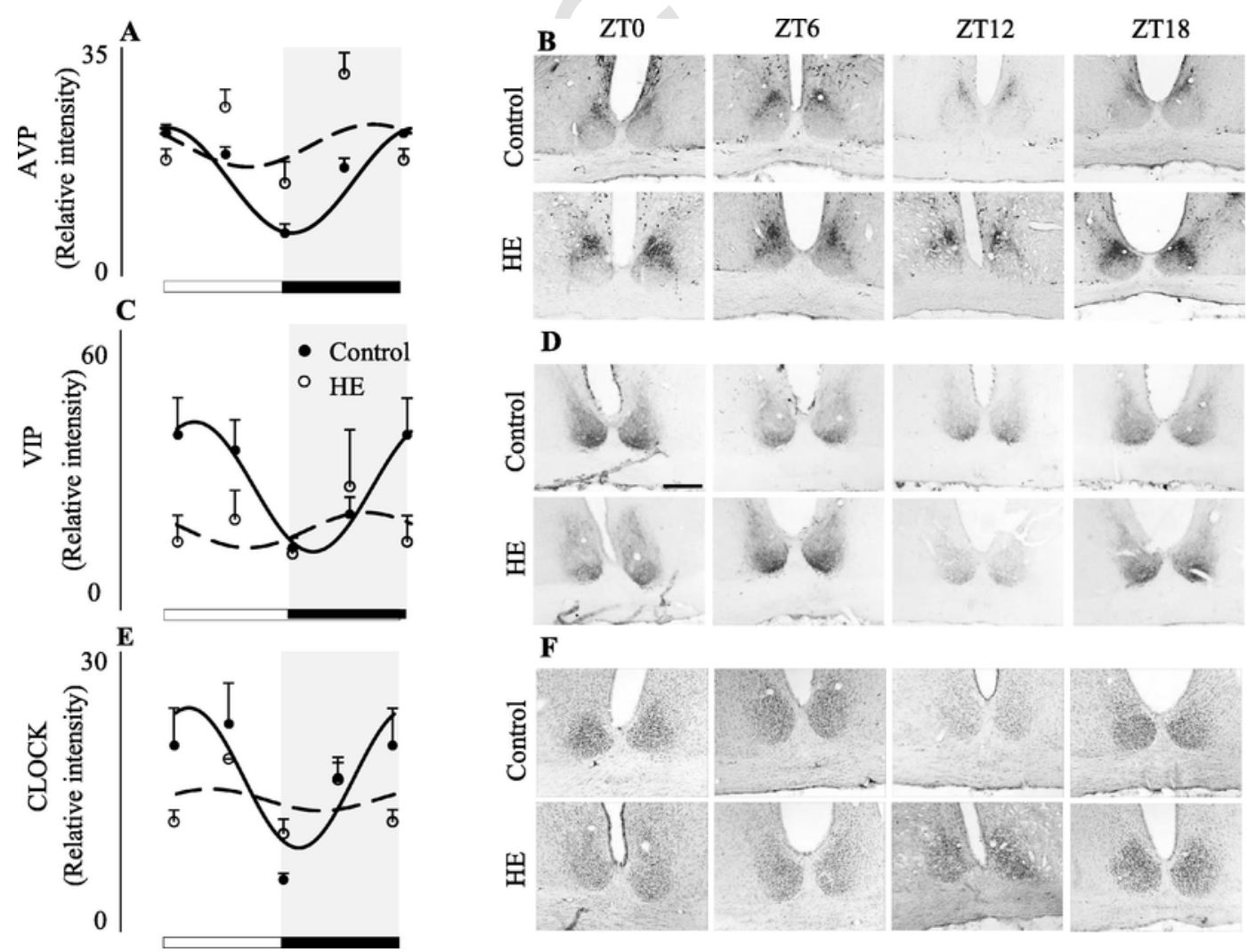

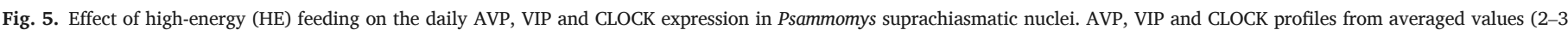

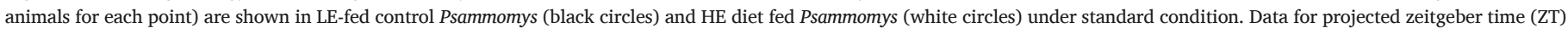

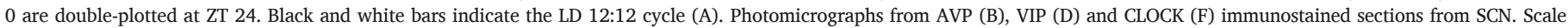
bar: $300 \mu \mathrm{m}$. Values are displayed as mean \pm SEM. Significant and non-significant cosinor regressions are shown with solid and dashed curves, respectively. 
$Z T 0$ - ZT12]. In the same way, cosinor analyses revealed significant daily oscillations for both VIP and CLOCK ( $<<0.05$; Fig. 6) with acrophases close to light on (Table 1) in the piriform cortex of control Psammomys fed with LE diet. Strikingly, as for the SCN, neither VIP (Fig. 6A and B) nor CLOCK (Fig. 6C and D) displayed significant daily variations in the cortex of HE-fed animals (Table 1). For both proteins, mean levels were kept at intermediate values compared to those in control animals.

\section{Discussion}

The present study highlights that diet-induced insulin resistance in Psammomys obesus leads to disturbances of brain clock processes, as evidenced by lengthening of the free-running period of wheel-running activity rhythm and impaired cycling of CLOCK and neuropeptides (AVP and/or VIP) in the master clock (SCN) and a secondary brain clock (piriform cortex).

\subsection{Diet-induced metabolic disturbances in Psammomys}

Staining of pancreatic insulin in control animals fed with low-energy diet displayed clear day-night variations with higher and lower contents at dawn and dusk, respectively. Previous data in rats have shown that insulin secretion undergoes daily variations (Kalsbeek and Strubbe, 1998), suggesting circadian control of both synthesis and release of insulin.

Caloric intake was identical between LE and HE group, this observation was already described in mice fed a high-fat diet ad libitumor with time-restriction (fed during active phase only) (Hatori et al., 2012).

Feeding Psammomys with calorie-enriched chow diet (HE) leads not only to higher body mass likely reflecting enhanced adiposity, but also to severe hyperinsulinemia. These symptoms are associated with increased insulin/glucose ratio, indicative of insulin resistance. The lower immunostaining of pancreatic insulin observed in HE-fed Psammomys at dawn is accompanied with elevated plasma insulin, which is the most significant parameter to indicate an aberrant metabolic state. Of note, insulinemia is constantly high in HE-fed animals, leading to the disappearance of day-night variations of plasma insulin. Despite such hyperinsulinemia, day-night levels of plasma glucose are not markedly increased in the HE-fed
Psammomys. It is expected that acute hyperglycemia occurs in these animals at least during postprandial periods.

Hatori team has indicated that high-fat diet blunts also the diurnal feeding rhythms under ad libitum access to food. This new feeding pattern perturbs metabolic pathways entrained by both circadian and feeding rhythms (Hatori et al., 2012).

\subsection{Consequences of diet-induced insulin resistance on temporal organization and exercise}

Psammomys fed with HE diet show an attenuation of daily rhythm of locomotor activity, especially during the active phase. An overall reduction of activity level is also observed under constant darkness. Similar dampened activity rhythms have been reported in high-fat-fed mice (Kohsaka et al., 2007) and high-fat-fed rats (Bravo et al., 2014). Beside the diminished locomotor activity during the active phase, high fat-fed rats also showed increased in locomotor activity during the rest phase (Bravo et al., 2014), a behavioral change not expressed by hyperinsulinemic Sand rats.

The elongation of the endogenous period of rest/activity rhythm in Psammomys under HE diet indicates an altered functioning of the master clock in the SCN. Of interest, comparable lengthening of the endogenous period has already been reported with various metabolic diseases, including diet-induced obesity in mice (Kohsaka et al., 2007), type 2 diabetes and obesity in $d b / d b$ mice (Grosbellet et al., 2016) and also insipidus diabetes in vasopressin-deficient rats (Li et al., 2009). The latter finding associated with AVP deficit raises the eventual involvement of vasopressinergic system in the central clock (see subSection 3.4).

\subsection{SCN organization, neuropeptides and CLOCK cycling in Psammomys}

Before discussing changes occurring in the SCN of HE-fed animals, we will first evoke the organization and neurochemistry of the Psammomys SCN, in relation to diurnality/nocturnality. Psammomys SCN exhibits the same neurochemical phenotype as that of other rodents, namely a dorso-medial region (shell) containing AVP-positive neurons and a ventral region (core) containing VIP-expressing neurons (Morin et al., 2006).
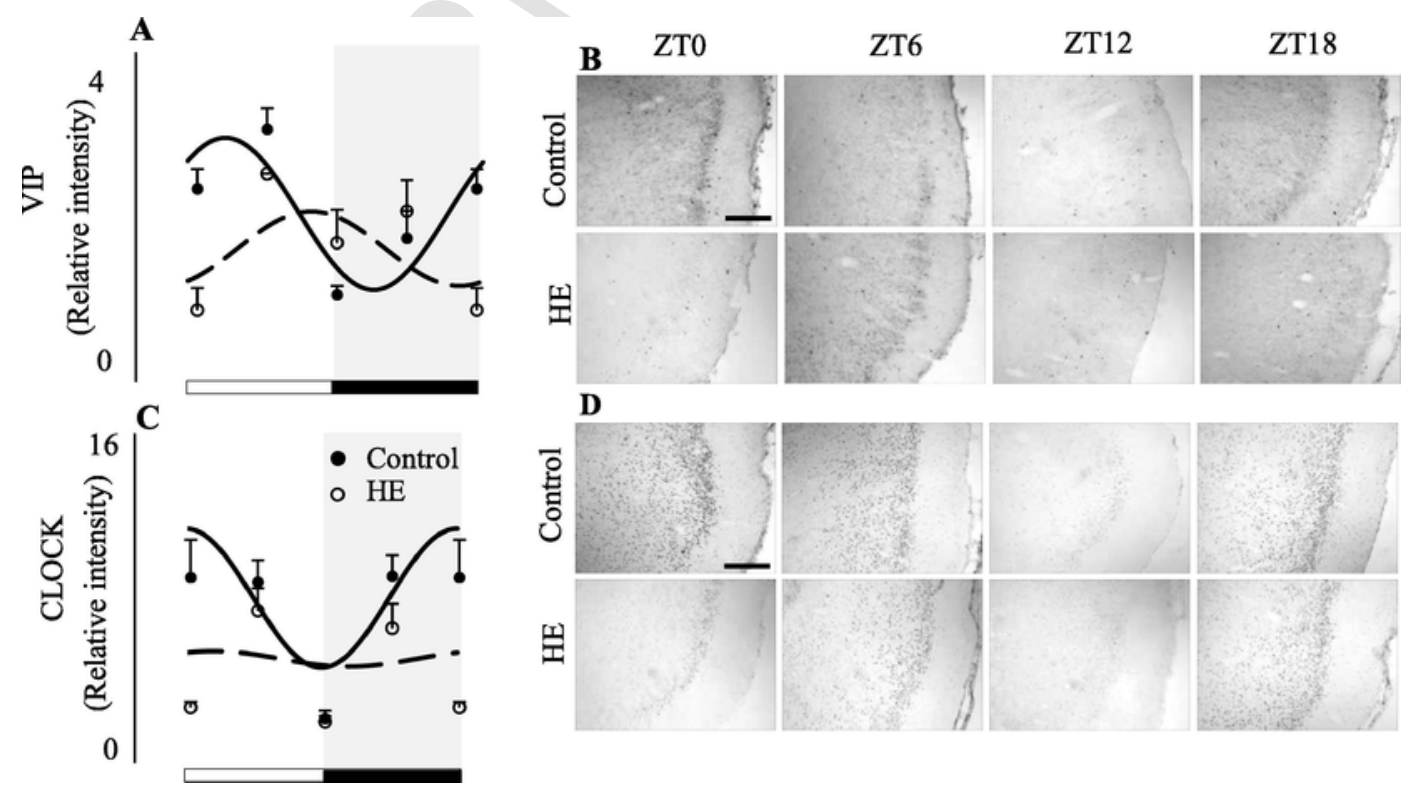

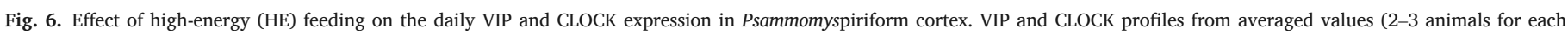

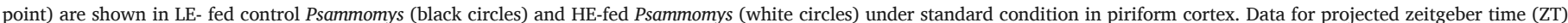

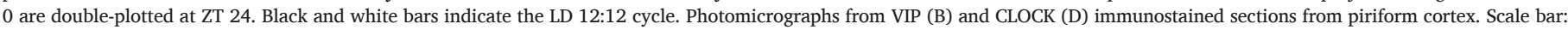
$250 \mu \mathrm{m}$. Values are displayed as mean \pm SEM. Significant and non-significant cosinor regressions are shown with solid and dashed curves, respectively. 
Under light-dark conditions, AVP and VIP content in the Psammomys SCN is strongly rhythmic, declining over the course of the light phase and gradually increasing until the end of the dark phase.

CLOCK is a circadian transcription factor critical for circadian clocks because CLOCK/BMAL1 dimers drive the main positive loop of the molecular clockwork. Here we found that CLOCK expression in the SCN show rhythmic variation in Psammomys.

This feature has already been described in diurnal rodents (Chakir et al., 2015), while CLOCK expression in the SCN is more generally constitutive in the SCN of nocturnal animals (Mendoza et al., 2007). Here we also noticed that the optic nerve and optic chiasm are much thicker when compared to those of nocturnal rodents, in line with previous observations done in another diurnal species, the Indian palm squirrel (Mammen and Jagota, 2011). Unexpectedly, our actimetry data reveal that most Psammomys have a nocturnal pattern of activity, while few individuals were even arrhythmic. Therefore, we suggest that the nocturnal pattern of activity expressed by the Psammomys studied here may secondarily be due to unidentified conditions of captivity (e.g., ambient humidity, dietary components, insufficient social cues or wheel access).

\subsection{SCN oscillations in high-energy-fed Psammomys}

Next, we sought to determine whether clock signaling may explain the altered endogenous period in HE-fed Psammomys. Interestingly, the calorie-enriched diet seems to alter CLOCK expression, leading to arrhythmicity in the SCN. This effect reveals that the molecular clockwork is somehow sensitive to metabolic changes associated with diet-induced insulin resistance. Although the endogenous period is lengthened in $d b /$ $d b$ mice, no significant changes are detected for PER1 and PER2 oscillations in their SCN (Grosbellet et al., 2016). Unfortunately, our trials for PER immunostaining in Psammomys were not successful, precluding any comparison in that respect.

High-fat feeding modifies circadian synchronization to light, the photic induction of both c-FOS and P-ERK in the suprachiasmatic nuclei was markedly reduced in mice (Mendoza et al., 2008). In addition the expression of many genes controlled by clock genes was found to be affected in the central clock of mice fed high-fat diet for 12 weeks (Cleal et al., 2014).

In mice, eating behavior was immediately affected by consumption of a high-fat diet; whereas, PER2 rhythms in the SCN were not affected by 1 week of high-fat diet consumption (Pendergast et al., 2013). In another study, period lengthening was observed after two weeks of high-fat diet (Kohsaka et al., 2007).

FOXO3-insulin signaling modulates the liver clock by reducing transcription of Clock gene (Chaves et al., 2014). Whether the same regulatory mechanisms take place in the SCN is not known yet, but they may give clues for explaining the dampened rhythm of CLOCK in hyperinsulinemic HE-fed animals.

The hypothesis of modified clockwork during diet-induced insulin resistance is further confirmed by the fact that two neuropeptides, VIP and AVP, which is a well-characterized clock-controlled protein, both lose their daily rhythmicity in the SCN of the same animals. A difference, however, distinguishes the effects for the two neuropeptides. While VIP expression loses its rhythmicity at intermediate control levels, AVP expression remains up-regulated in the SCN of HE fed animals throughout the daily cycle, suggesting possible alteration in SCN output rhythmicity. The elevated AVP levels in HE-fed Psammomys may reflect either up-regulated synthesis or attenuated release of AVP. Similar up-regulation of AVP expression in the SCN is observed in mice fed with an ultradian 6 -meals schedule ( 1 food access every $4 \mathrm{~h}$ ) to suppress the daily feeding/fasting cycle (Sen et al., 2016). This finding raises the possibility that changes in AVP expression are the consequences of an altered feeding pattern in Psammomys fed with HE diet. Further experiments are needed to address this issue.
In humans, the abnormal sympathetic over-activity reported during the rest phase is correlated to the extent of insulin resistance (Perciaccante et al., 2006). In the same context, reduced amplitude of locomotor activity rhythm in HE-fed Psammomys may be caused by altered SCN output that would deregulate daily variations of sympathetic tone. This hypothesis also deserves further investigation.

As mentioned in the Introduction, insulin signaling can modulate the SCN clock (Shibata et al., 1986) to maintain the robustness of peripheral cycling (Mori et al., 1985; Sakaguchi et al., 1988). Nevertheless, the present results suggest that circadian disturbances in hyperinsulinemic Psammomys might be related to reduced effects of insulin, possibly within the SCN clock.

The mechanism of hypothalamic insulin resistance induced by high-fat feeding results in local proinflammatory status, accompanied by impaired insulin signaling (i.e., increased serine phosphorylation of insulin receptor and insulin receptor substrate-2) (De Souza et al., 2005). Possible insulin resistance of circadian clocks in response to metabolic disorders would reduce sympathetic activity during the active phase and may improve glucose tolerance to favor possible metabolic adjustments on a short-term scale.

\subsection{Piriform cortex oscillations in high-energy-fed Psammomys}

The piriform cortex was chosen in the present study as a secondary brain oscillator. Moreover, the daily patterns of circadian genes in this structure are phased according to the rest/activity rhythm in several rodents (Ramanathan et al., 2010).

The piriform cortex in Psammomys exhibits daily variations of CLOCK and VIP with concomitant peaks around dawn. Strikingly, we found that both CLOCK and VIP rhythms are abolished during metabolic disturbances, as observed in the master clock of the SCN. Alterations of the molecular clock cycling under high-fat feeding were previously reported in the brainstem (Kaneko et al., 2009), the mediobasal hypothalamus and peripheral structures (Kohsaka et al., 2007).

Our findings extend this observation to circadian changes induced in the cerebral cortex during calories-enriched diet. Regarding the role of VIP in the cerebral cortex, previous works showed that VIP participates in the local control of energy metabolism by stimulating glycogenolysis (Magistretti et al., 1981).

It is tempting to speculate that the observed circadian disturbances in the piriform cortex may be caused in part by local insulin resistance. Alternatively, impaired rhythmicity of the central clock outputs may disturb SCN control of brain oscillators, such as the piriform cortex. More broadly, this hypothesis would also apply to disturbed SCN control of internal synchrony of peripheral clocks, through altered autonomic and hormonal rhythms.

\subsection{Conclusions}

In sum, it appears that in Psammomys, hyperinsulinemia is associated with abnormal clock molecular cycling, clock output release and altered regulation of circadian functions. Our findings on the clock responses to the diabetogenic consequences of calorie-enriched diet may help to elucidate the functional links between circadian clocks, insulin resistance and the circadian autonomic dysfunction.

\section{Experimental procedure}

\subsection{Animals}

All experiments were carried out in accordance with the European Union Directive (2010/63/EU). Thirty-one male Sand rats, Psammomys obesus (Harlan, Jerusalem, Israel), 6-month old, weighting $120-150 \mathrm{~g}$, were housed in individual cages in a controlled room at 22 $\pm$ 
$1{ }^{\circ} \mathrm{C}$ under a standard photoperiod of $12 \mathrm{~h}$ light/ $12 \mathrm{~h}$ dark cycle (12/12 LD, light onset defining ZT 0 at 7 AM, and light offset or ZT12 at 7 PM) with ad libitum access to food and tap water. White fluorescent tubes provided a light phase of 200 lux, while a constant dim red light $(<1$ lux) was on throughout the experiment. At their arrival, animals were fed for 3 weeks with the same LE diet as used by Harlan breeding colony (Table 2). Three weeks later, Psammomys were randomly assigned to one of two dietary groups, LE or HE diets, for three months.

The control group still consumed the same LE diet. The HE diet group received a standard laboratory chow which represents a HE regimen for Psammomys (leading to $14 \%$ increase in assimilable energy compared to the control LE diet; Table 2).

Body weight and food intake were measured weekly. Two months later, to assess rest/activity rhythm, animals were transferred to individual cages equipped with a running wheel and wheel-running activity was recorded every $5 \mathrm{~min}$ (Vitalview acquisition system, MiniMitter, Sunriver, OR, USA). Wheel-running activity was recorded under LD for 2 weeks then under constant darkness for 1 week and back to LD for 2 weeks. Several individuals ( $n=5$ and 4 in the LE and HE groups respectively) were arrhythmic and thus were discarded from actimetry and immunohistochemistry analyses.

The final number of rhythmic animals retained for the analysis was 12/17 animals in LE group and 10/14 animals for HE group. Thereafter, animals were euthanized at one of 4 times of day by overdose of sodium pentobarbital (i.p. $150 \mathrm{mg} / \mathrm{kg}$ ). Blood was collected by cardiac puncture just before intracardiac perfusion of paraformaldehyde.

\subsection{Tissue preparation and immunocytochemistry}

At four different times (i.e., ZT0, ZT6, ZT12 and ZT18), animals were perfused intra-cardially with $100 \mathrm{~mL}$ of $0.1 \mathrm{M}$ phosphate buffered saline (PBS, pH 7.4) followed by $400 \mathrm{~mL}$ of $4 \%$ paraformaldehyde in $0.1 \mathrm{M}$ phosphate buffer ( $\mathrm{pH}$ 7.4). Brains and pancreas were removed and postfixed in the same mixture for $24 \mathrm{~h}$.

\subsubsection{Brain}

Post-fixed brains were cryoprotected in $30 \%$ sucrose for $72 \mathrm{~h}$ and quickly frozen at $-45^{\circ} \mathrm{C}, 30 \mu \mathrm{m}$ coronal sections were cut at the rostro-caudal level of the SCN. Free-floating sections were processed overnight for the following primary antibodies: Vasopressin (Rabbit anti-AVP "Truus", 1:5,000; gift from Dr. Ruud M. Buijs, Netherlands Institute for Brain Research, Amsterdam, The Netherlands), Vasoactive Intestinal Peptide (Rabbit anti-VIP "Viper", 1:1000; gift from Dr. Ruud M. Buijs, Netherlands Institute for Brain Research, Amsterdam, The Netherlands), CLOCK (Goat anti-CLOCK, 1:1,000; SC-6927, Santa Cruz Biotechnology, Santa Cruz, CA, USA, Anti-CLOCK Registry AB_2082577 ), and Neuropeptide Y (Rabbit anti-NPY, 8184-4, 1:5,000, gift from Dr. Morton Møller, University of Copenhagen, Denmark) immunostaining

Table 2

Composition of low energy diet (LE) and high energy diet (HE).

\begin{tabular}{lllll}
\hline Diet & LE & & HE & \\
\hline & Weight & Calories & Weight & Calories \\
& 19.7 & 27.2 & 23.6 & 28.1 \\
Protein \% & 3.8 & 11.8 & 2.4 & 6.4 \\
Fat \% & 44.2 & 61.0 & 55.1 & 65.5 \\
Carbohydrate \% & 6.4 & - & 6.5 & - \\
Ash \% & 15.3 & - & 3.4 & - \\
Fiber \% & 10.6 & - & 9 & - \\
Moisture \% & & 2.9 & 3.4 & \\
Total energy & & & & \\
$\begin{array}{l}\text { (kcal/g) } \\
\text { Digestible nutrient }\end{array}$ & & 61.9 & 75.5 & \\
\% & & 1.8 & 2.5 & \\
$\begin{array}{l}\text { Digestible energy } \\
\text { (kcal/g) }\end{array}$ & & & & \\
\hline
\end{tabular}

overnight, followed by incubation with biotinylated goat anti-rabbit ( $2 \mathrm{~h}$; 1:500; Vector Laboratories, Burlingame, CA, USA, Goat Anti-Rabbit Registry $\left.A B \_2313606\right)$ or biotinylated rabbit anti goat $(2 \mathrm{~h} ; 1: 500$; Vector Laboratories) according to the primary antibodies. Then brain sections were rinced and incubated with a solution containing avidin-biotin-peroxidase complex (90 min; 1:500; Vectastain Elite ABC Kit, Vector, $\mathrm{ABC}$ Registry $A B_{-}$2336827) before substrate visualization using 3,3'-diaminobenzidine (Sigma-Aldrich, St Louis, MO, USA).

\subsubsection{Pancreas}

The pancreas samples were obtained from $\mathrm{n}=6$ individuals in both LE and HE ( $n=3$ at ZT0 and $n=3$ at ZT12). They were postfixed by overnight immersion in $4 \%$ paraformaldehyde before being dehydrated and embedded in paraffin blocks. Sixteen-micrometer pancreas adjacent sections were incubated with primary antibodies either against insulin (mouse monoclonal antibody, 1:60,000; clone HUI018 Novo Nordisk, Denmark) or somatostatin (mouse monoclonal antisomatostatin, 1:80,000; SOM018 Novo Nordisk, Denmark, Anti-Somatostatin Registry $A B_{-}$2315347), followed by incubation with secondary biotinylated donkey anti-mouse (1:2,000; Jackson Immunoresearch, West Grove, PA, USA, Donkey Anti-Mouse Registry AB_2307338) for one hour, then with Streptavidine peroxydase (1:2,000; Roche, Meylan, France) and revelation with diaminobenzidine. The specificity of all antibodies has been established previously by preadsorption control experiments. When tested by omission of primary antibodies, sections did not show any labeling (data not shown).

\subsection{Peroxidase staining quantification}

The optical density (OD) of AVP, VIP and CLOCK immunoreactivity was quantified using NIH Image $\mathrm{J}$ software (one or two sections per animal). The OD value resulted from the difference between the staining intensity in the SCN or cerebral cortex and the background intensity. The later was determined in an area of low density of cells in the same coronal section.

\subsection{Plasma assays}

Plasma glucose was measured using Biolabo Kit (LP80009; lot 031202A, Biolabo, France), and plasma insulin using Ultrasensitive rat insulin Elisa kit (\#90060, Crystal Chem Inc, IL, USA).

\subsection{Recording analysis}

ClockLab software (Actimetrics, Evanston, IL, USA) was used to analyze the daily rhythm of wheel-running activity using "Actogram" and "Activity profile" function, and daily period using "Chi-square periodogram" function.

\subsection{Statistical analysis}

Statistical analysis was performed by testing first the distribution of all variables for normality. Two-way analysis of variance on ranks with repeated measures was used to test the effect of diet and time in addition to the interaction between factors on body weight or caloric intake. Student's t test was then used for comparison of endogenous period and Kruskal-Wallis test for comparaison of levels of locomotor activity between the two diet groups. Two-way ANOVA on ranks test was performed to assess the effect of diet, time and the possible interaction between these two factors followed by Tukey-Kramer multiple comparison test on SCN or cortex markers and comparison of glycemia, insulinaemia and insulin/glycemia ratio between the two groups. Differences were considered significant at $\mathrm{P}<0.05$ (R software version 3.3.3). 
Rhythm characteristics defined by mean level A, amplitude B and acrophase $C$ were obtained by cosinor regression method $[y=A+B \cdot \cos (2 \pi \cdot(x-C) / 24)]$ using SigmaPlot software (SigmaPlot 12.5 software, Systat software Inc., San Jose, CA, USA) for neuropeptides (AVP, VIP) and clock proteins (CLOCK).

\section{Declaration of interest}

None.

\section{Conflicts of interest}

None.

\section{Contributors}

Touati conducted experimental part, collection, interpretation of data and the first drafting of the manuscript. Ouali-Hassenaoui participated in project management/ supervision, data interpretation and paper correction. Dekar-Madoui participated in analyzing data, correction of the paper. Challet contributed in the creation of research design, data interpretation and correction of manuscript. Pévet participated in data interpretation, final manuscript drafting and coordinated the overall study. Vuillez supervised the experimental part of the work, data interpretation and drafting the final version of manuscript. All authors have approved the final article.

\section{Acknowledgements}

We are indebted to S. Dumont and S. Gourmelen for hormonal assays and assistance with animal care, respectively. We also thank Dr. Sage-Ciocca (Chronobiotron UMS3415, CNRS and University of Strasbourg) for help with actimetry.

Funding: This work was supported by NEUROMED, FP7-REGPOT245807 (Capacities Programme in the European Union's $7^{\text {th }}$ Framework Programme 2011-2012); Ministry of High Education and Scientific Research-(Algiers). Experimental work was supported by CNRS and University of Strasbourg.

\section{Appendix A. Supplementary data}

Supplementary data associated with this article can be found, in the online version, athttps://doi.org/10.1016/j.brainres.2017.11.018.

\section{References}

Bravo, R., Cubero, J., Franco, L., Mesa, M., Galán, C., Rodŕguez, A.B., Jarne, C., Barriga, C., 2014. Body weight gain in rats by a high-fat diet produces chronodisruption in activity/inactivity circadian rhythm. Chronobiol. Int. 31, 363-370. https://doi.org/10. 3109/07420528.2013.859151.

Chakir, I., Dumont, S., Pévet, P., Ouarour, A., Challet, E., Vuillez, P., 2015. The circadian gene clock oscillates in the suprachiasmatic nuclei of the diurnal rodent barbary striped grass mouse, lemniscomys barbarus: a general feature of diurnality?. Brain. Res. 1594, 165-172. https://doi.org/10.1016/j.brainres.2014.10.063.

Challet, E., 2010. Interactions between light, mealtime and calorie restriction to control daily timing in mammals. J. Comp. Physiol. B. 180, 631-644. https://doi.org/10. 1007/s00360-010-0451-4.

Chaves, I., van der Horst, G.T., Schellevis, R., Nijman, R.M., Koerkamp, M.G., Holstege, F.C., Smidt, M.P., Hoekman, M.F., 2014. Insulin-foxo3 signaling modulates circadian rhythms via regulation of clock transcription. Curr. Biol. 24, 1248-1255. https://doi. org/10.1016/j.cub.2014.04.018.

Cleal, J.K., Shepherd, J.N., Shearer, J.L., Bruce, K.D., Cagampang, F.R., 2014. Sensitivity of housekeeping genes in the suprachiasmatic nucleus of the mouse brain to diet and the daily light-dark cycle. Brain Res. 1575, 72-77. https://doi.org/10.1016/j.brainres. 2014.05.031.
De Souza, C.T., Araujo, E.P., Bordin, S., Ashimine, R., Zollner, R.L., Boschero, A.C., Saad, M.J., Velloso, L.A., 2005. Consumption of a fat-rich diet activates a proinflammatory response and induces insulin resistance in the hypothalamus. Endocrinology 146, 4192-4199. https://doi.org/10.1210/en.2004-1520.

Emdin, M., Gastaldelli, A., Muscelli, E., Macerata, A., Natali, A., Camastra, S., Ferrannini, E., 2001. Hyperinsulinemia and autonomic nervous system dysfunction in obesity effects of weight loss. Circulation 103, 513-519. https://doi.org/10.1161/01.CIR.103. 4.513

Golombek, D.A., Rosenstein, R.E., 2010. Physiology of circadian entrainment. Physiol. Rev. 90, 1063-1102. https://doi.org/10.1152/physrev.00009.2009.

Grosbellet, E., Dumont, S., Schuster-Klein, C., Guardiola-Lemaitre, B., Pevet, P., Criscuolo, F., Challet, E., 2016. Circadian phenotyping of obese and diabetic $\mathrm{db} / \mathrm{db}$ mice. Biochimie 124, 198-206. https://doi.org/10.1016/j.biochi.2015.06.029.

Hackel, D.B., Frohman, L.A., Mikat, E., Lebovitz, H.E., Schmidt-Nielsen, K., Kinney, T.D., 1965. Review of current studies on effect of diet on the glucose tolerance of the sand rat (Psammomys obesus). Ann. N.Y. Acad. Sci. 131, 459-463. https://doi.org/ 10.1111/j.1749-6632.1965.tb34811.x.

Hatori, M., Vollmers, C., Zarrinpar, A., DiTacchio, L., Bushong, E., Gill, S., Leblanc, M., Chaix, A., Joens, M., Fitzpatrick, J., Ellisman, M., Panda, S., 2012. Time-restricted feeding without reducing caloric intake prevents metabolic diseases in mice fed a high-fat diet. Cell Metab. 15, 848-860. https://doi.org/10.1016/j.cmet.2012.04.019.

Kalsbeek, A., Strubbe, J.H., 1998. Circadian control of insulin secretion is independent of the temporal distribution of feeding. Physiol. Behav. 63, 553-560. https://doi.org/10. 1016/S0031-9384(97)00493-9.

Kaneko, K., Yamada, T., Tsukita, S., Takahashi, K., Ishigaki, Y., Oka, Y., Katagiri, H., 2009. Obesity alters circadian expressions of molecular clock genes in the brainstem. Brain. Res. 1263, 58-68. https://doi.org/10.1016/j.brainres.2008.12.071.

Kohsaka, A., Laposky, A.D., Ramsey, K.M., Estrada, C., Joshu, C., Kobayashi, Y., Turek, F.W., Bass, J., 2007. High-fat diet disrupts behavioral and molecular circadian rhythms in mice. Cell. Metab. 6, 414-421. https://doi.org/10.1016/j.cmet.2007.09. 006

Li, J.-D., Burton, K.J., Zhang, C., Hu, S.-B., Zhou, Q.-Y., 2009. Vasopressin receptor v1a regulates circadian rhythms of locomotor activity and expression of clock-controlled genes in the suprachiasmatic nuclei. Am. J. Physiol. Regul. Integr. Comp. Physiol. 296, R824-R830. https://doi.org/10.1152/ajpregu.90463.2008.

Magistretti, P.J., Morrison, J.H., Shoemaker, W.J., Sapin, V., Bloom, F.E., 1981. Vasoac tive intestinal polypeptide induces glycogenolysis in mouse cortical slices: a possible regulatory mechanism for the local control of energy metabolism. Proc. Natl. Acad. Sci. USA 78, 6535-6539, URL:http://www.pnas.org/content/78/10/6535.abstract.

Mammen, A.P., Jagota, A., 2011. Immunocytochemical evidence for different patterns in daily rhythms of VIP and AVP peptides in the suprachiasmatic nucleus of diurna Funambulus palmarum. Brain. Res. 1373, 39-47. https://doi.org/10.1016/j.brainres. 2010.12.018.

Mendoza, J., Lopez-Lopez, C., Revel, F., Jeanneau, K., Delerue, F., Prinssen, E., Challet, E., Moreau, J.-L., Grundschober, C., 2011. Dimorphic effects of leptin on the circadian and hypocretinergic systems of mice. J. Neuroendocrinol. 23, 28-38. https://doi.org/ 10.1111/j.1365-2826.2010.02072.x.

Mendoza, J., Pévet, P., Challet, E., 2007. Circadian and photic regulation of clock and clock-controlled proteins in the suprachiasmatic nuclei of calorie-restricted mice. Eur. J. Neurosci. 25, 3691-3701. https://doi.org/10.1111/j.1460-9568.2007.05626.x.

Mendoza, J., Pèvet, P., Challet, E., 2008. High-fat feeding alters the clock synchronization to light. J. Physiol. 586, 5901-5910. https://doi.org/10.1113/jphysiol.2008.159566.

Mori, T., Nagai, K., Hara, M., Nakagawa, H., 1985. Time-dependent effect of insulin in suprachiasmatic nucleus on blood glucose. Am. J. Physiol. Regul. Integr. Comp. Physiol. 249, R23-R30, URL:http://ajpregu.physiology.org/content/249/1/R23.

Morin, L., Shivers, K.-Y., Blanchard, J., Muscat, L., 2006. Complex organization of mouse and rat suprachiasmatic nucleus. Neuroscience 137, 1285-1297. https://doi.org/10 1016/j.neuroscience.2005.10.030.

Pendergast, J.S., Branecky, K.L., Yang, W., Ellacott, K.L.J., Niswender, K.D., Yamazaki, S., 2013. High-fat diet acutely affects circadian organisation and eating behavior. Eur. J. Neurosci. 37, 1350-1356. https://doi.org/10.1111/ejn.12133.

Perciaccante, A., Fiorentini, A., Paris, A., Serra, P., Tubani, L., 2006. Circadian rhythm of the autonomic nervous system in insulin resistant subjects with normoglycemia, impaired fasting glycemia, impaired glucose tolerance, type 2 diabetes mellitus. BMC. Cardiovasc. Disord. 6, 1-10. https://doi.org/10.1186/1471-2261-6-19.

Ramanathan, C., Stowie, A., Smale, L., Nunez, A.A., 2010. Phase preference for the display of activity is associated with the phase of extra-suprachiasmatic nucleus oscillators within and between species. Neuroscience 170, 758-772. https://doi.org/10.1016/j. neuroscience.2010.07.053.

Sakaguchi, T., Takahashi, M., Bray, G., 1988. Diurnal changes in sympathetic activity. relation to food intake and to insulin injected into the ventromedial or suprachiasmatic nucleus. J. Clin. Invest. 82, 282-286. https://doi.org/10.1172/JCI113584.

Sen, S., Raingard, H., Dumont, S., Kalsbeek, A., Vuillez, P., Challet, E., 2016. Ultradian feeding in mice not only affects the peripheral clock in the liver, but also the master clock in the brain. Chronobiol. Int. 34, 1-20. https://doi.org/10.1080/07420528. 2016.1231689.

Shibata, S., Liou, S.Y., Ueki, S., Oomura, Y., 1986. Inhibitory action of insulin on suprachiasmatic nucleus neurons in rat hypothalamic slice preparations. Physiol. Behav. 36, 79-81. https://doi.org/10.1016/0031-9384(86)90077-6.

Unger, J., McNeill, T., Moxley, R., White, M., Moss, A., Livingston, J., 1989. Distribution of insulin receptor-like immunoreactivity in the rat forebrain. Neuroscience 31, 143-157. https://doi.org/10.1016/0306-4522(89)90036-5. 